\title{
eCSM: Semantic Classification Model for Educational Repositories
}

\author{
Dongwon Jeong ${ }^{1}$, Myounghoi Choi ${ }^{1}$, and Keunhwan Jeon ${ }^{2}$ \\ ${ }^{1}$ Dept. of Informatics \& Statistics, Kunsan National University \\ San 68, Miryoung-dong, Gunsan, Jeollabuk-do, 573-701 Korea \\ \{djeong, cmh775\}@kunsan.ac.kr \\ ${ }^{2}$ Dept. of Internet Media Information, Kunjang College \\ 608-8, Seongsan-Myoen, Gunsan, Jeollabuk-do, 573-709 \\ khjeon@kunjang.ac.kr
}

\begin{abstract}
This paper proposes a new classification model for management of resources in an educational repository. To facilitate usability of educational resources, a proper classification model should be provided. If no classification model, it causes several issues such as inefficient management of educational resources, incorrect retrieval, and low usability. To achieve the goal of this paper, the model should support a classification mechanism in multiple viewpoints. The proposed model enables more precise and systematic management of implementation resources, and also increases the ease of usability.
\end{abstract}

Keywords: Semantic Classification, Educational Repositories, Classification Model.

\section{Introduction}

An educational repository is intended to manage efficiently and systematically a variety of resources generated in the educational course so that learning effects are maximized and qualitatively improved educational systems are developed. However, there have been few studies on educational repository system, especially, on classification scheme absolutely required to manage diverse and enormous educational resources and materials. An accurate classification scheme is required to store and manage systematically resources generated and to ensure accurate retrieval and maximized application.

Until now, in many other disciplines, multiple studies on classification schemes designed to manage efficiently and systematically their generated and used resources and apply correctly and exactly them have been conducted [1,2,3]. Although various classification schemes have been defined, most of them target commercial goods or services, and are inappropriate to the resource management for educational repository.

The classification scheme for educational resources is essentially similar to that for scientific technologies. Of the current scientific technology classification schemes in Korea, the most commonly used schemes are those suggested by Korea Science and Engineering Foundation (KOSEF) and Korea Research Foundation (KRF) $[4,5,6]$. 
These classification schemes pose the problem that they are based upon static classification models, i.e., static classification management models. In addition, a same subclass may be redundant with several other super-classes. Above all, since the relationship among the classes within a certain scheme is not clarified, it is difficult to interpret and understand their significance and implication, as well as impossible to define the associations among classification schemes and given careful consideration to the relationship between different resources. Importantly, if relying only on such classification schemes, it is impossible to classify and manage correctly and accurately the resources in an educational repository, because their classes were defined just from the viewpoint of scientific technologies. To the contrary, for resources to be managed in an educational repository, their classification scheme should be defined from the viewpoint of applications as well as simply scientific technologies [7].

In another aspect, the conventional classification schemes involve simple codebased classification models with tree structures or classification codes. In a codebased classification scheme, a super-class code (e.g., A10) becomes the prefix notation of its subclass code (e.g., A1011, A1012). Information - that is, the conceptions of classes, the relationship among the conceptions, integrity constraints, etc. - is expressed primarily rather implicitly than explicitly. For the reason, as any systematic processing is unavailable, guidelines are prepared and maintained merely via documentation. The above-mentioned problems of the logical models for code-based classification schemes may cause several problems including lack of consistency and flexibility attributed to classification criteria and levels mixed up in a same classification scheme [8].

The purpose of this paper is to define a classification scheme for educational repository and suggest a model designed to manage dynamically the defined classification scheme. A dynamic classification model, i.e., dynamic classification scheme management model enable add, merge and split operations in the defined scheme, and defining and managing the relationship between the classes to do so. In particular, it helps make classes significant and thereby define even the relationship among the classes, instead of defining the classes simply with codes in order to define and manage significantly the classification scheme.

\section{Definition of the Classification Model}

The matter of how to classify resources in an educational repository depending upon their generating subjects is of little importance. Regarding definition of classification model, one of the critically important characteristics of such the resources is that they may be generated for a wide range of disciplines. In addition, classification according to educational courses should be also taken into consideration. In this paper, classification according to developed language should be also considered owing to the nature of the specified resource range. First, let us give an example concerning educational course. "Database" and "Algorithm" courses should be definitely classified and each of them can be defined as a class. The resources generated in the individual courses become the resource objects belonging to the classes called either "database" or "algorithm". 
As a result, this paper supposes that in consideration of the three viewpoints, a resource classification scheme is to be defined. Fig. 1 shows the schematic diagram of a classification scheme considering the three viewpoints. Considering the three viewpoints suggests that an integrated classification scheme is completely composed of the three types of classification schemes. Further, it represents that a resource belongs to a certain class in the three different classification schemes according to the three viewpoints. In particular, the viewpoint of applications can be regarded as that of application user. In other words, it indicates the viewpoint for users who are familiar with or want to search and apply data under different classification schemes - e.g., "personal information management system" or "video management system" - from scientific technology classification scheme or language classification scheme. Defined in this paper, all the three viewpoints for classification are intended not to meet the universal needs for all applied fields, but to perform a case paper concerning the said multiple viewpoints and dynamic classification model for the purpose of this paper. In this light, the classification viewpoints and classes for each viewpoint should be defined by target fields. However, the concept of the classification scheme management, and its basic architecture and operations suggested by this paper are applicable to all kinds of fields.

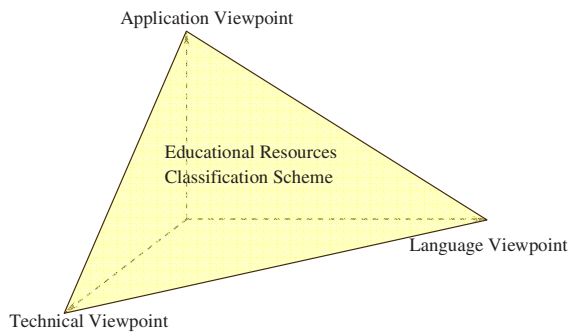

Fig. 1. Classification scheme \& its multiple viewpoints

The purpose of this paper is to define a classification model for educational repository resources, named eCSM (Educational Classification Scheme Management Model), and eCSM is defined as follows:

Definition 1. A classification scheme for resources R, called eCSM consists of 5tuple $<\mathrm{R}, \mathrm{C}, \mathrm{C}_{\mathrm{R}}, \mathrm{P}, \mathrm{OP}>$, where

- $\mathrm{R}$ : the group of target resources to be classified. Composed of 3-tuple $<\mathrm{R}_{\text {source }}, \mathrm{R}_{\text {doc }}$, Ru_guide $>$, where

- $R_{\text {source }}$ : Actually implemented resources, i.e., implemented sources

- $\mathrm{R}_{\mathrm{doc}}$ : Reporter on and interface with implemented resources

- $\mathrm{R}_{\mathrm{u} \_ \text {guide: }}$ User guide covering implementation settings, installation and so on.

- C: All classification schemes; $C=\left\{C_{S} \cup C_{A} \cup C_{L}\right\}$, where in order denoting schemes for science technology, application, and language viewpoint.

- $\mathrm{C}_{\mathrm{R}}$ : Root classes of each classification scheme; $\mathrm{C}_{\mathrm{R}}=\left\{\mathrm{c}_{\mathrm{RS}}, \mathrm{c}_{\mathrm{RA}}, \mathrm{c}_{\mathrm{RL}}\right\}$, where each element indicates the root class of $\mathrm{C}_{\mathrm{S}}, \mathrm{C}_{\mathrm{A}}$, and $\mathrm{C}_{\mathrm{L}}$. 
- P: P represents the relationships between classes and the suggested classification scheme has not a tree structure but a graph model.

- OP: The set of operations depending on changes in the classes. Operations are classified largely into the operations for classes, $\mathrm{OP}_{\text {class }}$ and the operations related to resource allocation depending on changes in the classes, $\mathrm{OP}_{\text {instance. }}$

In Definition 1, resources $\mathrm{R}$ is grouped by classification scheme $\mathrm{C}$, while the relationship among the classes corresponding to elements of $\mathrm{C}$ is expressed by $\mathrm{P}$. If a new class is added, the values $\mathrm{C}, \mathrm{R}$ and $\mathrm{P}$ are modified by an operation of $\mathrm{OP}$.

In order to implement a more intelligent and versatile classification scheme, the inter-class relationship or class-resource relationship should be defined. Relationship means the relation between two entities. In this paper, largely three types of relationship exist. Additionally, the term relationship stated in this paper is used as the same meaning with Predicate or Property in other similar research fields. This paper doesn't define all the significant relationships available in the selected target field. If required in future, the relationship can be defined and applied in a dynamic manner. The types of relationship defined in this paper include threes - inter-class (class-class) relationship, class-resource relationship and inter-resource (resource-resource) relationship. In eCSM, the relationship P is defined as Definition 2.

Definition 2. Assuming that the groups of inter-class, class-resource and interresource relationships are expressed as PCC, PCR and PRR, respectively, the relationship P of eCSM consists of 3-tuple $<$ PCC, PCR, PRR $>$.

First, inter-class relationship means the relationship between two classes in the classification scheme and its typical examples include parentOf(superClassOf) and chil$\mathrm{dOf}($ subClassOf). Class-resource relationship is a simple relation of is_a, which represents that resources belong simply to the relevant terminal classes and are subject to grouping. This paper targets primarily the short-term project outputs generated in the departments of computer science, in universities or colleges. Accordingly, the generated resources include outcomes of development (implemented data, programming sources), reporters, and user guides such as development settings. Thus, there are the inter-resource relationship, for example, sourceOf, documentOf and userGuideOf. Additionally, the proposed classification model can define new types of relationship, other than the above-mentioned ones.

Fig. 2 illustrates the case where the above-mentioned relationship exists. $c_{R}$ represents the parent class of $\mathrm{c}_{1}$; if the direction is reversed, the meaning is also reverse. That is, there seems to be the relationship between $c_{4}$ and $c_{2}$ that the former is the child class of the latter. It is seen that there is just is_a relationship between the class and the resource. For inter-resource relationship, $r_{1}$ represents the user guide of $r_{2}$, which suggests that the two resources are the outputs from a same short-term project.

OP represents the group of operations depending on changes in the classes and is divided largely into the operations for classes and the operations for resource reallocation depending upon changes in the classes. The followings are the main operations for the classes. 
- GENERATE: Generate a new class. This operation is associated with other operations such as ADD, GENERALIZE, MERGE and SPLIT. GENERATE operation is divided into twos: one is GENERATE operation (associated directly with ADD or GENERALIZE) without any change in the existing classes and the other is the operation (associated with MERGE or SPLIT) with any change in the existing classes.

- ADD NODE: Add a class as either terminal node or non-terminal node after GENERATE operation.

- ADD RELATIONSHIP: Add the relationship between classes.

- MERGE: Merge classes according to the attributes of newly generated or existing classes. Integrate classes with similar attributes and if merging terminal classes, their resources also belong to the new class.

- GENERALIZE: Generalize subclasses to a single super class, unlike MERGE.

- SPLIT: Split in details depending upon the attributes of class.

- DELETE: Delete the classes which have failed to be merged or split.

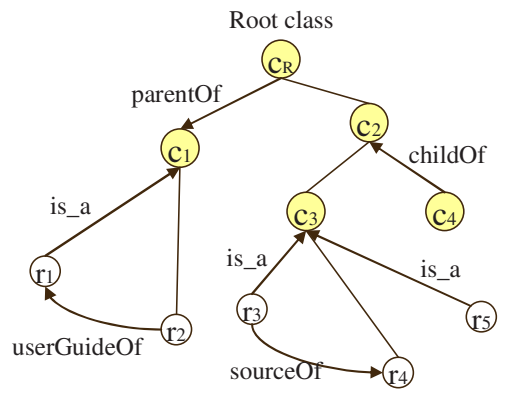

Fig. 2. An example of the expression of relationship in eCSM

\section{Metamodel for Data Management in eCSM}

Fig. 3 shows a metamodel intended to implement eCSM. View defines the information on the viewpoint, and Classification_Class is used to define each class in the classification scheme. All the groups of Classification_Class involving a certain View form a classification scheme derived from the viewpoint. Thus, in this paper, the followings can be defined substantially.

Fig. 4 illustrates the case where class-resource relationship and inter-resource relationship are substantially expressed. In this figure, the resource with resource_ID of "R0101" belongs to class "C0001" and the relationship is "is_a". This relationship is expressed in PCR. The inter-resource relationship is expressed in PRR, which represents the relationship that resource "R0101" is the source to implement another resource "R1100".

There are many ways to define physically a class and an instance, and their relationship. Typically, the methods of defining them with a programming language or with description language such as XML [9], RDF [10] or DAML [11] are used. Recently, OWL (Web Ontology Language)-based approaches have attracted public 


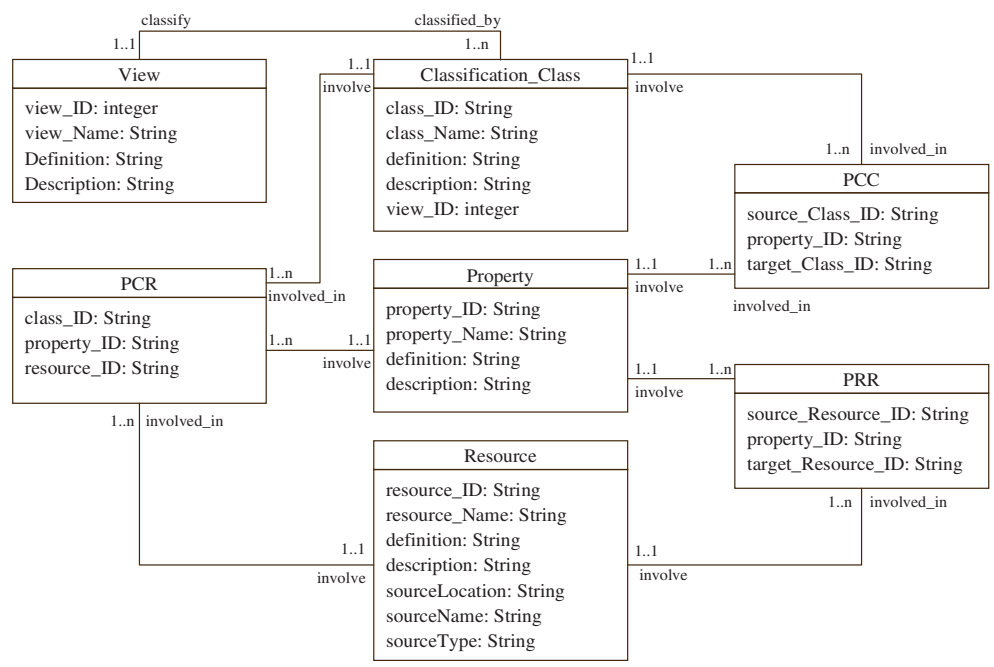

Fig. 3. Metamodel intended to implement eCSM

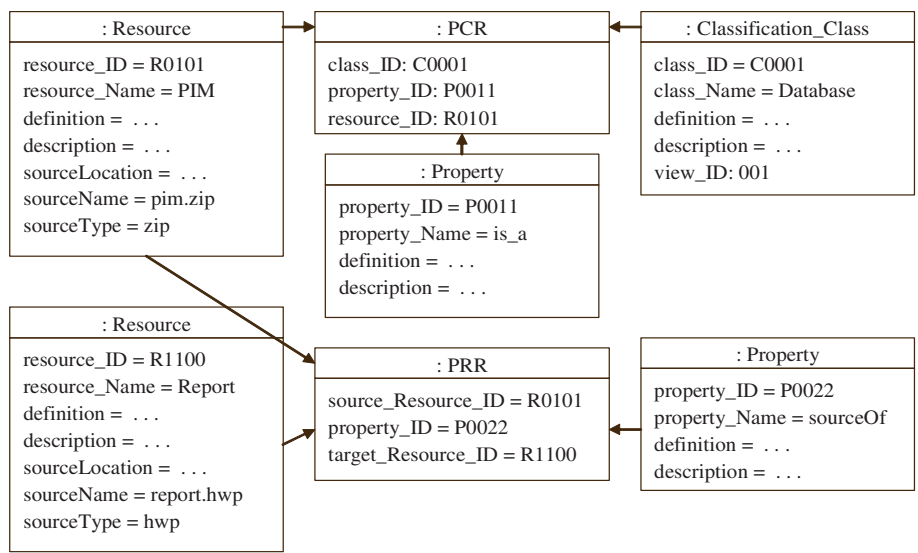

Fig. 4. An example of relationship expression

attention. OWL is a more intelligent language than XML and has been recognized as a next-generation description language integrating RDF or DAML [12,13].

\section{Implementation Prototyping of eCSM}

Fig. 5 shows the system architecture intended to develop an educational repository system based on eCSM.

Resource Management Agent is responsible for user authentication and interfacing including resource registration and retrieval. If the user registers a resource, Relationship Management Agent generates and adds its relationship. 
Once registration event occurs, first Relationship Management Agent generates the inter-resource relationship, and subsequently, the class-resource relationship is automatically generated through the index table located in Resource Management Agent according to the classification information selected by the user. Any additional relationship, that is, relationship which cannot be automatically generated is manually generated by the administrator. In this paper, the phrase "automatically generated" means not that the system automatically generates and manages classes, but that the resource-class relationship is spontaneously generated and managed on the basis of the classification information defined by the user (customized) as well as the system resources.

As one of the most important modules, Classification Class Management Agent plays the role of generating and managing the classification scheme. Various operations (GENERATE, ADD, SPLIT, MERGE, GENERALIZE and DELETE) are performed. Once an event concerning class operations occurs, Relationship Management Agent generates or modifies the inter-class relationship. Further, if class-resource relationship is to be changed, the relevant jobs are performed. In connection with these jobs, some operations not affecting the relationship with resources can be automatically executed, while other operations affecting the relationship are difficult to execute automatically. For the reason, they are either manually operated or semiautomatically performed via an efficient interface.

At last, Storage Management Agent stores information through interlocking with database, and if needed, delivers appropriate information to other individual agents. Interlocking with database can be readily implemented by means of database connector such as ODBC or JDBC, which is already a well-known technique.

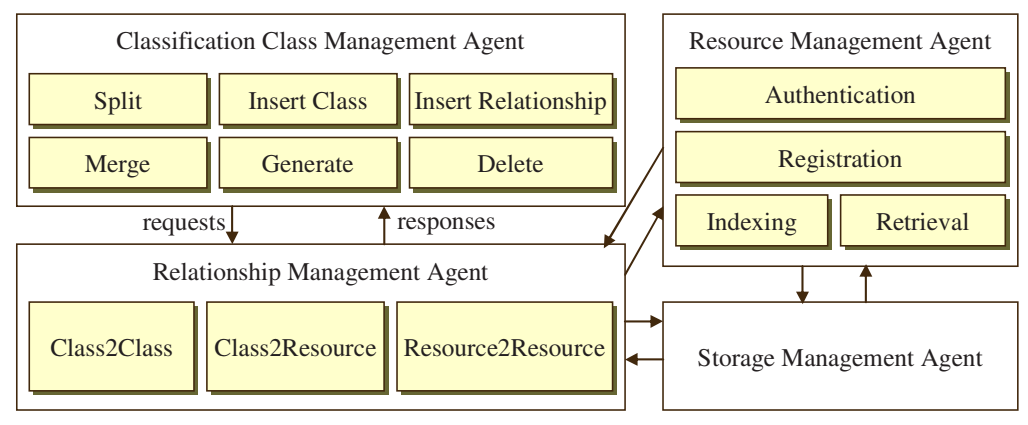

Fig. 5. System Architecture for eCSM-based educational repository system

As previously mentioned, this paper targets short-term projects in computer science field at the level of university or college. The purpose of this paper is to develop a classification model capable of managing dynamically and defining a classification scheme from various viewpoints in order to ensure more efficient management of educational repository resources. Thus, this paper doesn't attempt to define a perfect classification scheme for educational repository. Instead, it attempts to define partially the classification scheme as required for prototype development. 


\section{Conclusion}

This paper suggested eCSM, a classification model designed to manage consistently resources in an educational repository system and to improve their applications. Furthermore, it analyzed the science technology classification scheme adopted by KOSEF and Korea Research Foundation and defined additionally some classes in order to develop an appropriate classification scheme for the management of educational repository resources. Especially, the suggested eCSM takes multiple viewpoints - i.e., of users (applications) and of languages as well as simply of scientific technologies - into consideration, and thus, facilitates applications among the users. In addition, it defined how to manage dynamically classification scheme and the involved main operations, and the model to be implemented with the classification scheme, and presented the results of implementation.

The proposed eCSM first suggests an efficient resource management model intended to develop an educational repository management system. In order to manage and use efficiently resources, an efficient classification scheme and its management are absolutely essential. eCSM comes up with a solution for this requirement. Further, it can not only function as the fundamentals for developing a classification scheme for the still poorly studied field of educational resource management, but also facilitate to develop a better educational repository management system.

Further studies need to define more perfect classification schemes in discussion and collaboration with many researchers and experts engaging in the relevant fields. Further, they need to analyze fully the limitations of the suggested eCSM by expanding the target range and thereby developing more practical systems.

\section{References}

1. UNDP (United Nations Development Programme), United Nations Standard Products and Service Code, White Paper, http://www.unspsc.org (2001)

2. Harmonized System Committee, Harmonized System Convention (2004)

3. UNDP (United Nations Development Programme), Better Supply Management with UNSPSC, White Paper, http://www.unspsc.org (2006)

4. KRF (Korea Research Foundation), http://www.krf.re.kr/ (2006)

5. KOSEF (Korea Science and Engineering Foundation), http://www.kosef.re.kr/ (2006)

6. KISTI (Korea Institute of Science and Technology Information) (2006)

7. KCS (Korean Chemical Society), http://newkcsnet.kcsnet.or.kr/ (2006)

8. Kim, D., Lee, S.-g., Chun, J., and Choi, D.-H.: A Semantic Classification Model for eCatalogs," Korea Information Science Society, Journal of KISS: Databases, Vol. 33, No. 1 (2006) 102 116

9. Yergeau, F., Cowan, J., Bray, T., Paoli, J., Sperberg-McQueen, C.M., and Maler, E.: Extensible Markup Language (XML) 1.1, W3C Recommendation, W3C (2004)

10. Beckett D.: RDF/XML Syntax Specification (Revised), W3C Recommendation, W3C (2004)

11. The DARPA Agent Markup Language, About DAML, http://www.daml.org/ (2003)

12. McGuinness, D.L., and van Harmelen F.: OWL Web Ontology Language Overview, W3C Recommendation, W3C (2004) 